\title{
Side population cells separated from A549 lung cancer cell line possess cancer stem cell-like properties and inhibition of autophagy potentiates the cytotoxic effect of cisplatin
}

\author{
YANG YANG $^{1 *}$, YUXIA FAN $^{2 *}$, YU QI $^{1}$, DONGLEI LIU $^{1}, \mathrm{KAI} \mathrm{WU}^{1}$, FENGBIAO WEN $^{1}$ and SONG ZHAO ${ }^{1}$ \\ Departments of ${ }^{1}$ Thoracic Surgery and ${ }^{2}$ Thyroid Surgery, The First Affiliated Hospital, Zhengzhou University, \\ Zhengzhou, Henan 450052, P.R. China
}

Received March 6, 2015; Accepted May 5, 2015

DOI: $10.3892 /$ or.2015.4057

\begin{abstract}
Recent studies have suggested that cancer stem cells (CSCs) may be responsible for tumorigenesis and contribute to resistance to chemotherapy. Side population (SP) cells are thought to be enriched for CSCs in most types of human tumors. Therefore, the aim of the present study was to sort SP cells using an A549 lung cancer cell line, identify the cancer stem cell-like properties of SP and determine the role of autophagy in the survival of SP cells of lung cancer. SP cells were isolated by fluorescence-activated cell sorter (FACS) from A549 lung cancer cells, and the CSC-like properties were verified through confocal fluorescence imaging, sphere formation assays, cell proliferation and colony formation assay, gene expression in vitro and tumor formation in vivo. The role of autophagy in the survival of SP cells was assessed by western blotting and flow cytometric analysis. A549 lung cancer cells contained $1.10 \%$ SP cells. SP cells showed higher abilities of sphere and colony formation, cell proliferation and self-renewal. Moreover, compared to non-SP, SP cells demonstrated a higher mRNA expression of stem cell markers (MDR1, ABCG2 and OCT-4). The clone formation efficiency of SP cells was significantly higher than that non-SP cells under the same conditions. Expression of autophagosomes in SP cells was markedly lower than that in non-SP cells. However, the level of autophagy in SP cells was found to be markedly increased in the presence of cisplatin. In addition, inhibition of autophagy enhanced the effects of apoptosis induced by cisplatin. SP cells from the A549 lung cancer cell line possessed the properties of CSCs and were used to investigate the further characteristics of lung CSCs. SP cells were
\end{abstract}

Correspondence to: Dr Song Zhao, Department of Thoracic Surgery, The First Affiliated Hospital, No. 1 East Jianshe Road, Zhengzhou University, Zhengzhou, Henan 450052, P.R. China

E-mail: zhaosong_thoracic@126.com

*Contributed equally

Key words: lung cancer, side population cell, cancer stem cell, autophagy, chemoresistance more resistant to chemotherapy and inhibition of autophagy enhanced the effects of apoptosis induced by the chemotherapeutic agent, cisplatin. These results may provide insight into novel therapeutic targets.

\section{Introduction}

Although advances have been made in decreasing the mortality rates, the prognosis of lung cancer remains poor. With an overall 5-year survival rate of $<18 \%$, the poor prognosis of lung cancer is mainly due to lack of understanding of the mechanisms involved in oncogenesis and drug resistance to chemotherapy (1). Recent findings suggest that cancer stem cells (CSCs) may be responsible for tumorigenesis and contribute to resistance to chemotherapy $(2,3)$. In those studies, it was found that tumors are organized in hierarchical heterogeneous cell populations, which constitute a spectrum of phenotypically different cell types. Among these cell subsets, CSCs have the ability to sustain tumor growth in a very small fraction of tumor cells and possess the characteristics of longevity, self-renewal, multilineage differentiation capacity and tumorigenicity. In addition, CSCs express high levels of the adenosine-triphosphate-binding cassette (ABC) transporter that is responsible for pumping out chemotherapeutic drugs (4). Thus, identification of CSCs may be useful to understand the mechanism of generation of lung cancer and identify more effective therapeutic methods.

A useful method for the isolation of CSCs is dependent on their ability to discharge lipophilic and fluorescent dyes such as Hoechst 33342 by the ABC membrane transporter. Based on the capacity to efflux Hoechst 33342 dye, side population (SP) cells are considered to be enriched for CSCs in most types of human tumors, such as glioma (5), pancreatic cancer (6), renal cell carcinoma (7), breast cancer (8), and nasopharyngeal carcinoma (9). Moreover, accumulating evidence shows that SP cells were described to possess cancer stem cell-like properties and may be a useful tool to identify cancer stem cell populations $(10,11)$. However, to the best of our knowledge, few studies have examined the role of SP cells in lung cancer.

Autophagy is a highly conserved catabolic process among all eukaryotes involving the degradation of cellular organelles 
and proteins. In a previous study, we found that autophagy acted as a potential survival mechanism when the cancer cells were treated with chemotherapy, and inhibition of autophagy enhanced the lethal effect of cisplatin (12). In addition, recent evidence has indicated that several types of tumor are dependent on autophagy for growth under normal conditions (13). However, the exact role of autophagy in the survival of SP cells of lung cancer to chemotherapy has yet to be determined.

In the present study, we isolated SP cells from an A549 lung cancer cell line using a fluorescence-activated cell sorter (FACS). The cancer stem cell-like properties of SP cells were verified through confocal fluorescence imaging, sphere formation and cell proliferation and colony formation assays, and gene expression in vitro and tumor formation in vivo. We also investigated whether autophagy is involved in the survival of SP cells in lung cancer cell lines to cisplatin.

\section{Materials and methods}

Cell culture. A549 lung cancer cells were obtained from the Key Laboratory of The First Affiliated Hospital of Zhengzhou University, and were cultured in RPMI-1640 supplemented with $100 \mathrm{ml} / 1$ fetal bovine serum (FBS) and $100 \mathrm{U} / \mathrm{ml}$ penicillin/streptomycin at $37^{\circ} \mathrm{C}$ in a humidified atmosphere of $95 \%$ air and $5 \% \mathrm{CO}_{2}$.

FACS analysis and purification of SP cells. A549 cells were eluted with $2.5 \mathrm{~g} / 1$ trypsin and $0.5 \mathrm{~g} / 1$ ethylenediaminetetraacetic acid, centrifuged, washed and resuspended at $1 \times 10^{6}$ cells $/ \mathrm{ml}$ in pre-warmed RPMI-1640 containing $2 \% \mathrm{FBS}$. The cells were then incubated for $120 \mathrm{~min}$ at $37^{\circ} \mathrm{C}$ with $5 \mu \mathrm{g} /$ $\mathrm{ml}$ Hoechst 33342 alone or in combination with $50 \mu \mathrm{g} / \mathrm{ml}$ verapamil which inhibited ABC transporters. After the incubation process, the cells were washed in phosphate-buffered saline (PBS), centrifuged at $4^{\circ} \mathrm{C}$ and resuspended in PBS solution supplemented with $2 \% \mathrm{FBS}$ and $1 \mathrm{mM}$ HEPES. The cells were filtered through a $40 \mu \mathrm{m}$ mesh filter and reserved at $4^{\circ} \mathrm{C}$ for flow cytometry analysis. The SP cells were selected and sorted by FACS (BD Biosciences, San Jose, CA, USA). The Hoechst dye was excited with a UV laser at $346 \mathrm{~nm}$ and its fluorescence was measured with 630/22 (Hoechst 33342 Red) and 424/44 filters (Hoechst Blue).

Confocal fluorescence imaging. A549 cells were washed, resuspended and incubated with $5 \mu \mathrm{g} / \mathrm{ml}$ Hoechst 33342 in the dark at $37^{\circ} \mathrm{C}$ for $30 \mathrm{~min}$. The cells were washed again in PBS three times, and $400 \mu 1$ PBS was added. Confocal microscopy was used to capture the images of SP cells.

Sphere formation assays. SP cells were seeded at a density of $1 \times 10^{2}$ cells/well in 6-well plates. The cells were cultured in Dulbecco's modified Eagle's medium (DMEM)/F12 medium containing $10 \mathrm{mg} / \mathrm{l}$ insulin, $20 \mu \mathrm{g} / \mathrm{l}$ epidermal growth factor (EGF) and $10 \mu \mathrm{g} / 1$ basic fibroblast growth factor (bFGF). Insulin, EGF and bFGF were added every 3 days. After culture for 10-14 days, the formation of floating spheres was visually assayed. The spheres containing $>30$ cells were selected, and then trypsinized to gain the single cells. After suspension of these cells, the cells were added into 6-well plates at a density of $1 \times 10^{2}$ cells/well. The secondary sphere formation was assayed after culture for 10-14 days. The number of spheres was counted under the dissecting microscope.

Cell proliferation assay. SP and non-SP cells were counted and added into 96-well plates at a density of $10^{4}$ cells/well. The cells were cultured in RPMI-1640 supplemented with $100 \mathrm{ml} / 1$ FBS for 5 days. A Cell Counting kit-8 (CCK-8) analysis was used to detect and compare the growth of these cells each day. The growth curve was plotted according to the absorbance of each well.

Colony formation assay. SP and non-SP cells were cultured in RPMI-1640 supplemented with $100 \mathrm{ml} / \mathrm{l} \mathrm{FBS}$ at a density of $1 \times 10^{2}$ cells/well for 10-14 days. The number of clones that contained $>50$ cells were counted and recorded.

Gene expression. Total RNA was extracted from SP and non-SP cells separately using TRIzol reagent according to the manufacturer's instructions. RNA was reverse-transcribed into cDNA using the SuperScrit First-Strand Synthesis system (Invitrogen, Carlsbad, CA, USA) as described in instructions. The RT-PCR was carried out using the SuperScript One-Step kit (Invitrogen). The primers used were: MDR1, 5'-CCCATC ATTGCAATAGCAGG-3' and 5'-GTTCAAACTTCTGCTCC TGA-5' for a 157-bp fragment; ABCG2, 5'-CTGAGATCCTGA GCCTTTGG-3' and 5'-TGCC CATCACAACATCATCT-3' for a 380-bp fragment; OCT4, 5'-CTGTAACCGGCGCCAGAA-3' and 5'-TGCATGGGAGAGCCCAGA-3' for a 218-bp fragment; GAPDH, 5'-ACCACAGTCCATGCCATCAC-3' and 5'-TCC ACCACCCTGTTGCTG TA-3' for a 249-bp fragment. The PCR products were separated by electrophoresis in $2 \%$ agarose gel.

Tumor formation. SP and non-SP cells were sorted and resuspended at a density ranging from $10^{4}$ to $10^{3}$ cells. The cells were mixed with $50 \mu 1$ Matrigel to prevent cell dispersion and loss. The cells were subcutaneously injected into 6- to 7-week-old nude mice, obtained from the Experimental Animal Center of Zhengzhou University, China. The mice were monitored to assess tumor formation for 8 weeks. The experiments were approved by the Laboratory Animal Ethics Committee of Zhengzhou University.

Autophagy expression. Cells were stained with $0.05 \mathrm{~mol} / 1$ of monodansylcadaverine (MDC) solution for $15 \mathrm{~min}$ and observed using a confocal laser microscope to detect the autophagolysosomes. The cells were collected and lysed in RIPA buffer containing protease inhibitor and phosphatase inhibitor. Lysated proteins were centrifuged at $14,000 \mathrm{rpm}$ for $10 \mathrm{~min}$ and quantified. Proteins were separated by a $4-20 \%$ gradient SDS/PAGE gel and transferred to polyvinylidene fluoride (PVDF) membranes. The membrane was placed in TBST solution containing 5\% non-fat skim milk at room temperature for $1 \mathrm{~h}$. The membranes were incubated with diluted primary antibodies overnight at $4^{\circ} \mathrm{C}$ and washed with TBST three times for $10 \mathrm{~min}$. Subsequently, the membrane was reacted with secondary antibodies in $2.5 \%$ non-fat skim milk for $1 \mathrm{~h}$ at room temperature, followed by washing with TBST three times for $10 \mathrm{~min}$. Blots were stripped and re-blotted with anti- $\beta$-actin, and the membrane was examined for the band. 

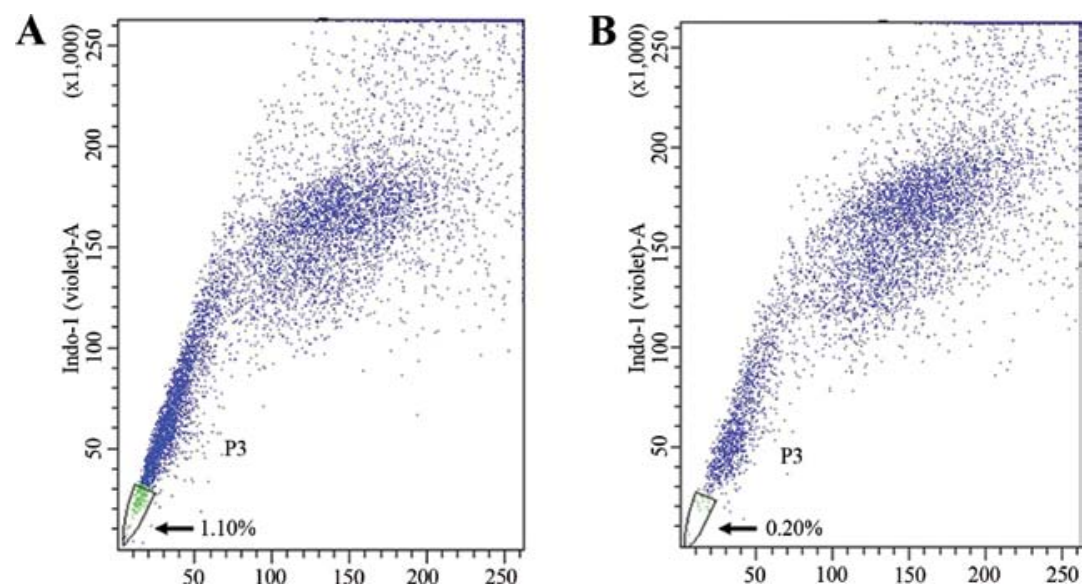

Figure 1. Hoechst 33342 dye staining profiles of A549 lung cancer cells. SP cells were isolated and analyzed by FACS. The proportions of SP cells from A549 lung cancer cells in the absence or presence of verapamil were (A) $1.10 \%$ and (B) $0.20 \%$, respectively. SP, side population.

A

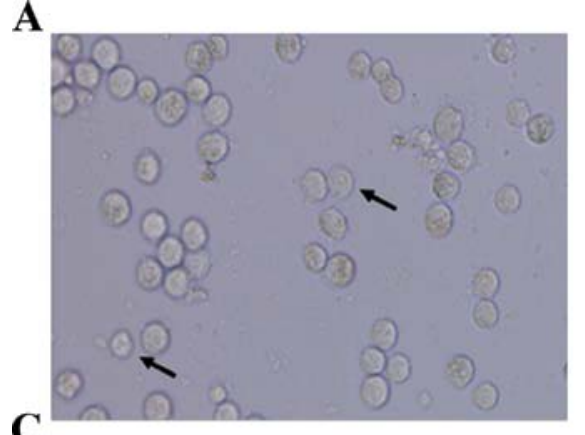

$\mathbf{C}$

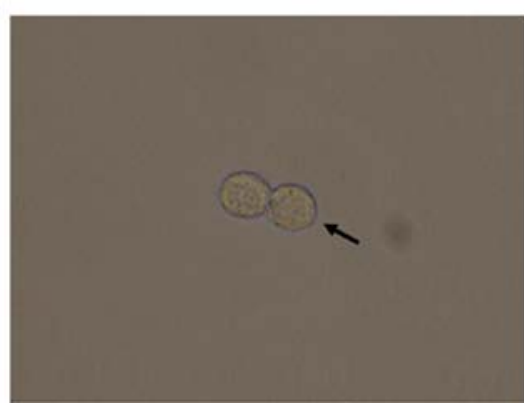

B

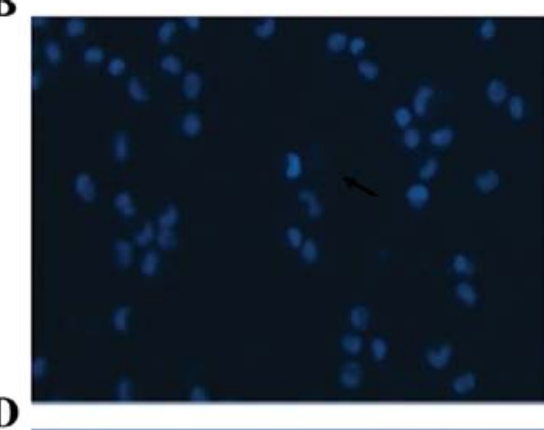

D

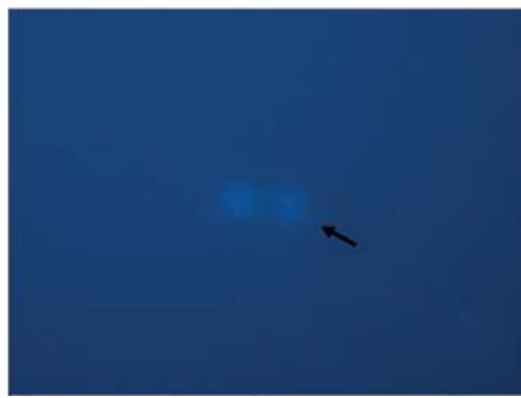

Figure 2. Identification of SP cells. SP cells (arrows) were not stained by Hoechst 33342 dye. Hoechst 33342 dye staining profiles of A549 lung cancer cells were observed using a confocal microscope in usual [(A) magnification, x100 and (C) magnification, x200); and [dark-field (B) magnification, x100 and (D) magnification, $\mathrm{x} 200]$. SP, side population.

Apoptosis assay. SP cells were treated with the chemotherapeutic drug cisplatin for $24 \mathrm{~h}$ and then incubated with or without 3-methyladenine (3-MA), which is an inhibitor of autophagy. Autophagy expression in cells treated with chemotherapeutic agent was measured as mentioned above. The level of apoptosis of SP cells with or without 3-MA was determined by flow cytometric analysis. The cells were then incubated in $5 \mathrm{ml}$ binding buffer containing $5 \mathrm{ml}$ Annexin $\mathrm{V}$ and $5 \mathrm{ml}$ propidium iodide. The cells were gently vortexed and assayed with an Annexin V-FITC apoptosis detection kit according to the manufacturer's instructions.

Statistical analysis. Data were presented as the mean \pm SD. Statistical significance $(\mathrm{P}<0.05)$ was evaluated by the Student's t-test.

\section{Results}

Identification of SP cells in lung cancer. SP cells were separated from A549 lung cancer cells by their fluorescence profiles in a dual wavelength analysis by FACS. The characteristic tails isolated from the complete population were identified.

The percentage of SP cells analyzed by FACS was $1.10 \%$ (Fig. 1A). After preincubation with verapamil which inhibited $\mathrm{ABC}$ transporters for $90 \mathrm{~min}$, the percentage of SP cells decreased to $0.20 \%$ (Fig. 1B). This result showed that Hoechst 33342 exclusion was verapamil-sensitive. The images of SP cells were captured by confocal microscopy (Fig. 2).

Sphereformation. The floating spheres of SPcells were observed after 3-4 days of seeding, and the primary spheres containing 

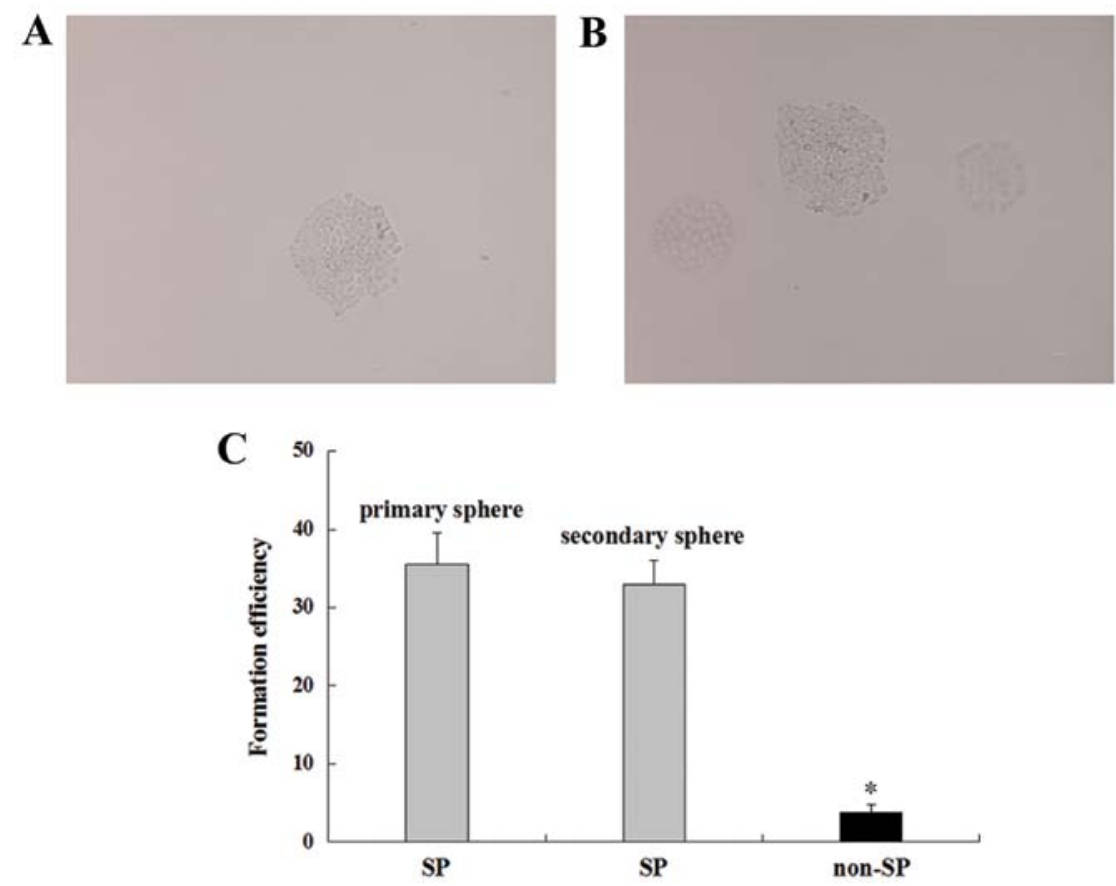

Figure 3. Sphere forming capability of A549 lung cancer SP cells. Representative micrographs showed the size of tumor floating sphere by the SP cells after 3-4 days (A) magnification, x100 and 10-14 days (B) magnification, x100. (C) There was a statistically significant difference between SP and non-SP cells in the formation efficiency of primary and secondary sphere $\left({ }^{*} \mathrm{P}<0.05\right)$. However, for SP cells there was no statistically significant difference in the formation of primary and secondary spheres (C). SP, side population.
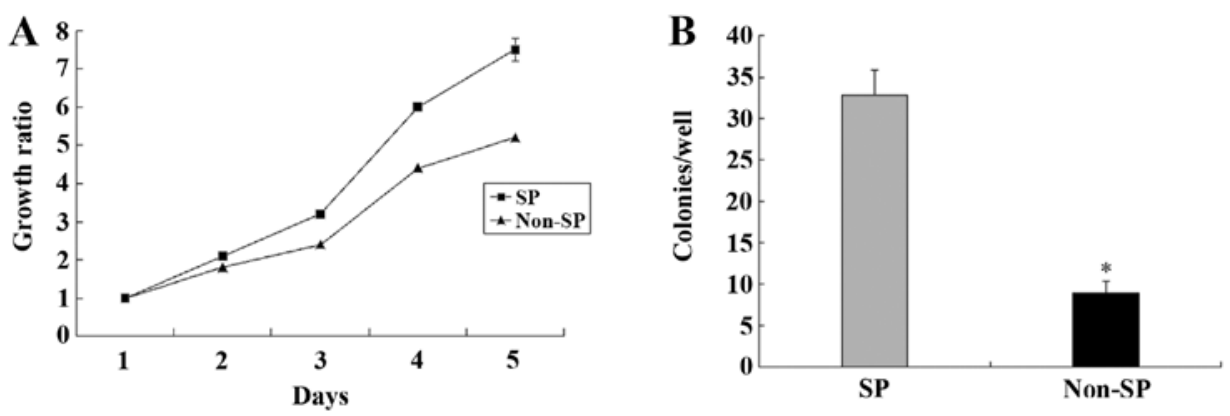

Figure 4. Cell growth curve and colony formation. (A) SP cells had a higher proliferative ability than non-SP cells ( $\mathrm{P}<0.05)$. (B) The clone formation ability of $\mathrm{SP}$ cells was significantly higher compared to non-SP cells with an equal cell number $\left({ }^{*} \mathrm{P}<0.05\right)$. SP, side population.

$>30$ cells were observed after 10-14 days (Fig. 3A and B). The amount and formation speed of the secondary sphere were similar to those of the primary sphere (Fig. 3C), suggesting that SP cells have self-renewal ability. However, non-SP cells could not be propagated under the same conditions.

Cell proliferation and colony formation. We performed CCK-8 analysis to compare the cell proliferation of SP with non-SP cells. A significant difference in cell proliferation between SP and non-SP cells was observed after 5 days of culture $(\mathrm{P}<0.05)$ (Fig. 4A). In addition, in the colony formation assay, there was a statistically significant difference between SP and non-SP cells over 10-14 days $(\mathrm{P}<0.05)$ (Fig. 4B).

Expression of stem cell genes. MDR1 and ABCG2, which belong to $\mathrm{ABC}$ transporters, contribute to the chemoresistance of SP cells. Compared to non-SP cells, we found that the expression of MDR1 and ABCG2 in SP cells was significantly

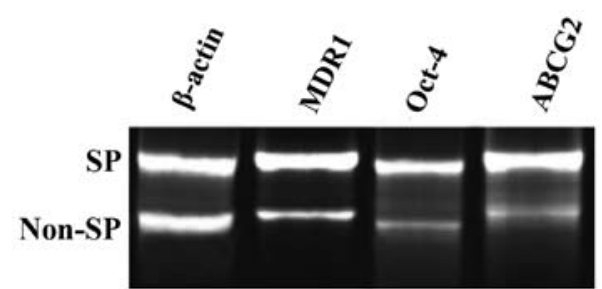

Figure 5. Expression of stem cell markers in SP and non-SP cells. The expression of MDR1, ABCG2 and OCT-4 in SP cells was significantly higher than that in non-SP cells, respectively. There were significant differences between $\mathrm{SP}$ and non-SP cells $(\mathrm{P}<0.05)$. SP, side population.

upregulated. OCT-4, an embryonic stem cell biomarker, was also highly expressed in SP compared with non-SP cells. The results indicated that SP cells from A549 lung cancer cells had some features of cancer stem cells (Fig. 5). 


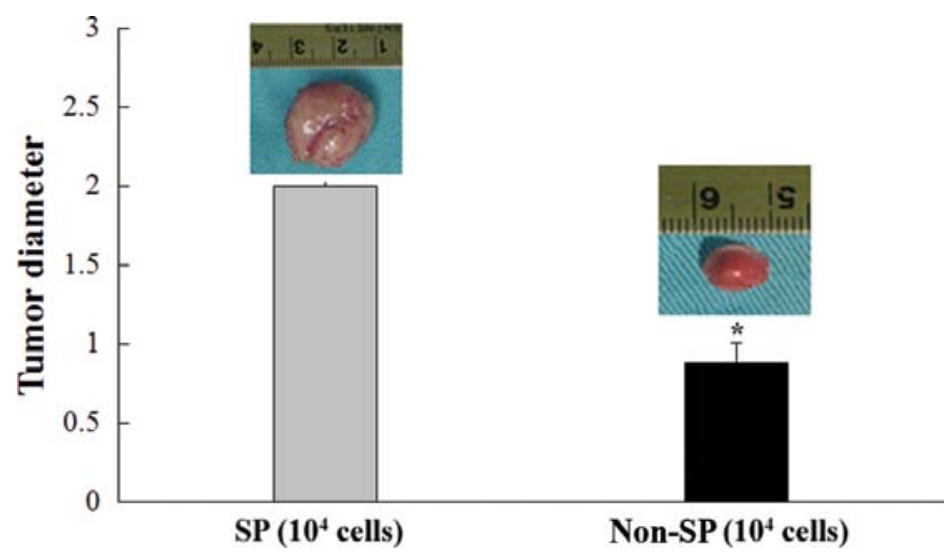

Figure 6. Tumorigenic potential of SP cells and non-SP cells in vivo. The tumor diameter of SP cells was significantly higher than that of non-SP cells with an equal cell number ( $\mathrm{P}<0.05$ vs. SP cells). SP, side population.
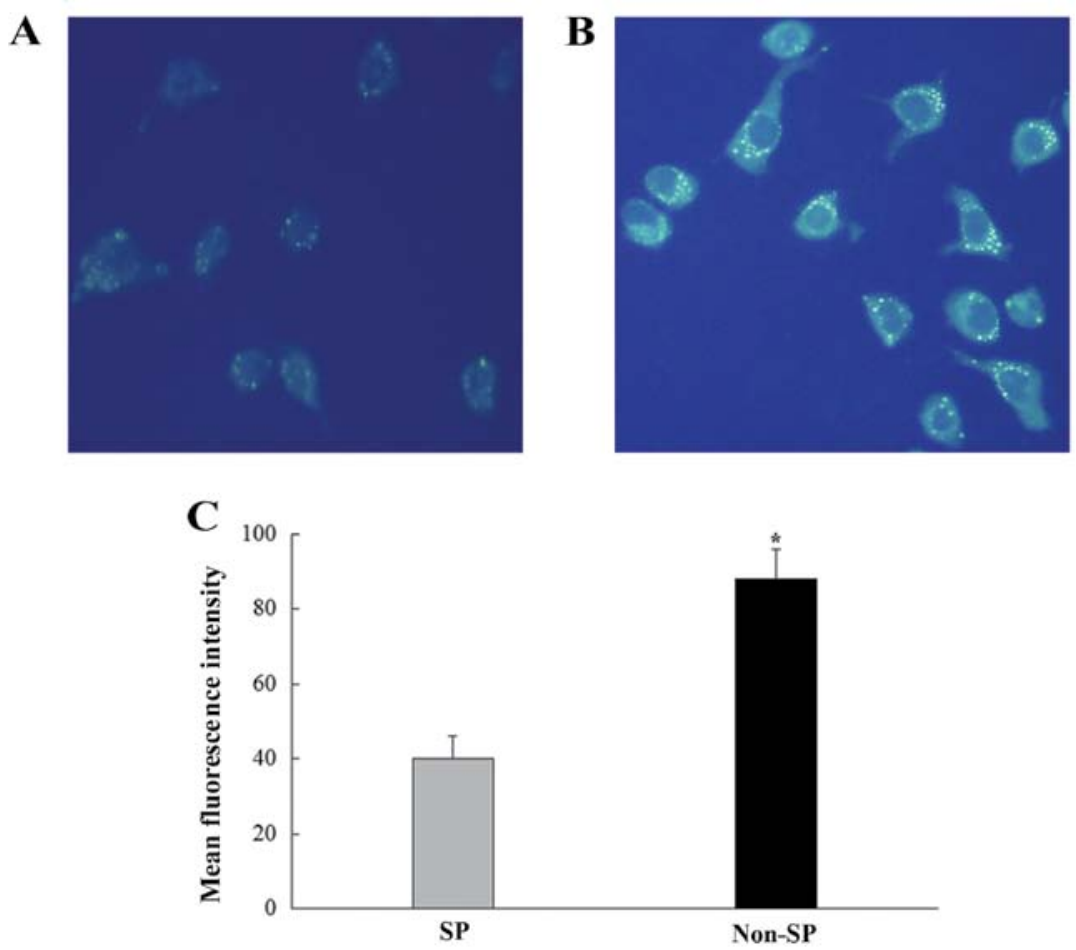

Figure 7. Autophagy expression in SP and non-SP cells. The cells were stained with MDC, and the autophagolysosomes were observed using a confocal laser microscope. The expression of autophagolysosomes in SP cells [(A) magnification, x200] was significantly less than that in non-SP cells [(B) magnification, $\mathrm{x} 200]$. (C) There was a statistically significant difference of mean fluorescence intensity between SP and non-SP cells ("P $<0.05)$. SP, side population; MDC, monodansylcadaverine.

Tumor formation. To determine the tumorigenic potential of lung cancer SP cells in vivo, SP and non-SP cells were subcutaneously injected into 6- to 7-week-old nude mice. Each mouse was injected with different density of SP and non-SP cells individually. After 8 weeks, almost all of the mice injected with SP cells at densities of $10^{4}-10^{3}$ cells formed tumors, whereas a smaller number of mice injected with non-SP cells at a density of $10^{4}$ cells formed tumors. Additionally, the tumor diameters were apparently smaller than those in SP cells with an equal cell number (Fig. 6).

Autophagy expression in SP and non-SP cells. MDC staining was performed to detect the autophagolysosomes. We observed that the number of autophagolysosomes in SP cells was less than that in non-SP cells by confocal laser microscopy (Fig. 7). The expression of Beclin-1 and LC3-II, both of which were involved in the autophagic process, was lower in SP cells than that in non-SP cells (Fig. 8).

Autophagy provides resistance against chemotherapy in $S P$ cells. A significant increase in autophagic response towards the chemotherapeutic drug, cisplatin, was observed in SP cells. Autophagosomes were found to be markedly increased in the presence of cisplatin. Compared to SP cells without treatment of cisplatin, the expression of Beclin-1 and LC3-II in SP cells treated with cisplatin was markedly upregulated. In addition, 


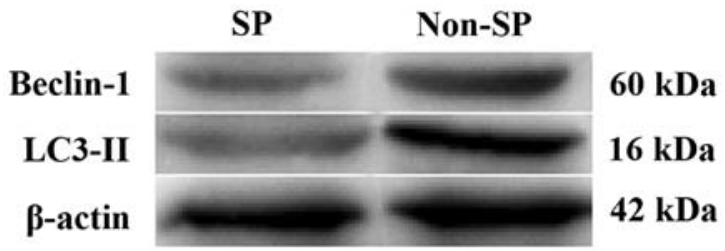

Figure 8. Western blot analyses of Beclin-1 and LC3-II in SP and non-SP cells. Compared to non-SP cells, the expression of Beclin-1 and LC3-II cells were significantly lower in SP cells $(\mathrm{P}<0.05)$. The result showed that the SP cells had lower autophagic activity than non-SP cells. SP, side population.

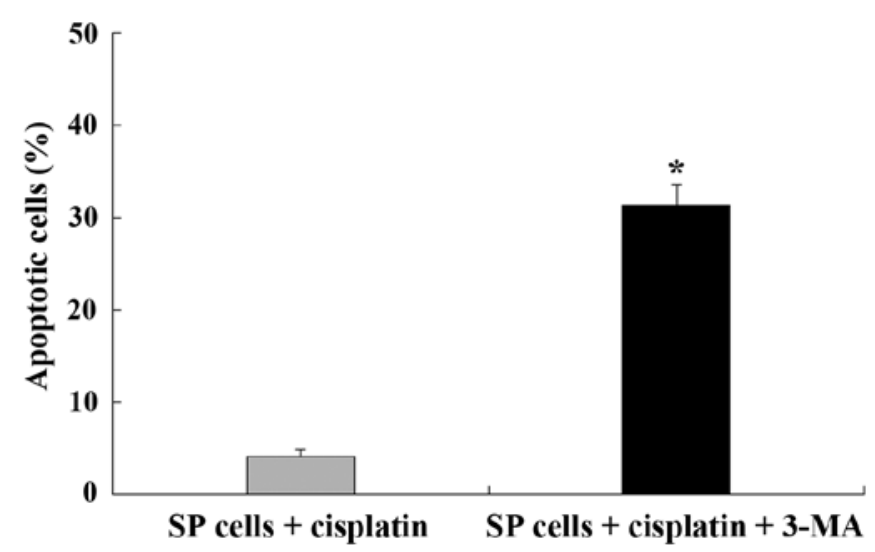

Figure 9. Inhibition of autophagy enhances the effects of apoptosis induced by cisplatin. The rate of apoptosis in SP cells with cisplatin was $(4.2 \pm 0.7) \%$, while it increased up to $(31.4 \pm 2.3) \%$ in the presence of 3 -methyladenine (3-MA), an inhibitor of autophagy. Significant difference of the two groups $\left({ }^{*} \mathrm{P}<0.05\right)$. SP, side population.

the rate of apoptosis in SP cells with cisplatin was $(4.2 \pm 0.7) \%$, while it increased up to $(31.4 \pm 2.3) \%$ in the presence of 3-MA, which is an inhibitor of autophagy (Fig. 9).

\section{Discussion}

The CSCs hypothesis suggests that cancer may be maintained by CSCs that possess the properties of self-renewal, multipotency and carcinogenesis (14). Recent evidence has shown that CSCs are the root of recurrence, metastasis and resistance to the chemotherapy of cancer $(15,16)$. Therefore, it is suggested that targeting CSCs in cancer provides a novel effective therapeutic strategy to eradicate them completely. Regarding the methods of isolating CSCs, identification of specific markers for CSCs is the ideal method. It is reported that CSCs have been identified by specific markers in several solid tumors, including breast (17), prostate (18) and colon cancer (19), and melanoma (20). However, specific markers of CSCs in malignant tumors remain to be identified. Furthermore, the so-called 'CSCs', which had differentiated surface markers, were not considered real CSCs, as they had already differentiated $(21,22)$.

Due to the absence of specific markers of CSCs in most malignant tumors, sorting SP cells using FCM by a dye Hoechst 33342 has become the main method of investigating CSCs. SP cells efflux the dye Hoechst 33342 through an ABC membrane transporter which is responsible for chemoresistance and is also thought to possess stem cell characteristics in several types of cancer (23). In the present study, we isolated
SP cells from the A549 lung cancer cell line by flow cytometry and then verified the cancer stem cell-like properties of SP cells in vitro and in vivo.

It is reported that CSCs have an anchorage-independent growth ability and sphere formation assays can be used to check the self-renewal ability of cells in vitro (24). In the present study, the formation of the primary and secondary floating sphere showed that SP cells isolated from A549 lung cancer cells possessed self-renewal ability. Infinite proliferation and tumorigenic abilities are notable features of CSCs (25). Compared to non-SP cells, the fact that single cells became anchorage-independent more rapidly and formed colonies showed that SP cells had malignant and tumorigenic abilities. These findings suggest that SP cells from lung cancer cells possessed CSCs-like properties.

The expression of MDR1 and ABCG2, which belong to the ABC transporter superfamily, were upregulated in SP cells when compared with that in non-SP cells. This result was consistent with the higher chemotherapeutic resistance of SP cells. OCT-4 is a transcriptional activator belonging to the POU family, and it is also an important regulator of selfrenewal and differentiation in stem cells. The overexpression of these representative stem cell genes in SP cells suggested that the SP cells characterized the properties of CSCs.

For the in vivo experiments, SP and non-SP cells were injected into nude mice subcutaneously. SP cells formed tumors after 8 weeks at densities of $10^{3}$ and $10^{4}$, whereas non-SP cells only formed tumors in some mice at a density of $10^{4}$, and the tumors were much smaller than that of the SP group. Of note was that some non-SP cells also formed tumors in some nude mice, suggesting that although CSCs were enriched in SP cells, non-SP cells also contain some CSCs.

Autophagy is a pivotal process for cell survival by decomposing unessential proteins or impaired cell organelles and utilizing them as energy when cells suffer from malnutrition, exposure to toxic materials, hypoxia and virus infection (26). If the stresses continue, this process leads to autophagic cell death (27). Notably, autophagy has two contradictory aspects in that it acts as a mechanism of cell survival and death (28). In a previous experiment, using A549 lung cancer cells, we identified that inhibition of autophagy was capable of promoting the apoptosis induced by chemotherapeutics. In the present study, we found that the expression of autophagosomes was much lower in SP cells than that in non-SP cells. In addition, Beclin-1 and LC3-II, both of which were involved in the autophagic process, also showed a lower expression in SP cells compared to that in non-SP cells. However, the autophagy level was found to be markedly increased in the presence of cisplatin and inhibition of autophagy enhanced the effects of apoptosis induced by the chemotherapeutic agent, cisplatin, in lung cancer SP cells. Thus, autophagy is a potential therapeutic target for lung cancer.

In conclusion, SP cells from the A549 lung cancer cell line can be effectively sorted using FACS with Hoechst 33342. SP cells possess the properties of CSC, such as increased proliferation, higher clonogenicity, and stronger tumorigenicity. Moreover, SP cells are more resistant to chemotherapy and inhibition of autophagy enhances the effects of apoptosis induced by cisplatin. Therefore, these findings collectively suggest that SP cells from the A549 lung cancer cell line have 
cancer stem cell-like properties and autophagy may be an attractive therapeutic target for human lung cancer.

\section{References}

1. Siegel R, Ma J, Zou Z and Jemal A: Cancer statistics, 2014. CA Cancer J Clin 64: 9-29, 2014.

2. O'Flaherty JD, Barr M, Fennell D, Richard D, Reynolds J, O'Leary J and O'Byrne K: The cancer stem-cell hypothesis: Its emerging role in lung cancer biology and its relevance for future therapy. J Thorac Oncol 7: 1880-1890, 2012.

3. Richard V, Sebastian P, Nair MG, Nair SN, Malieckal TT, Santhosh Kumar TR and Pillai MR: Multiple drug resistant, tumorigenic stem-like cells in oral cancer. Cancer Lett 338 300-316, 2013.

4. Gillet JP, Efferth T and Remacle J: Chemotherapy-induced resistance by ATP-binding cassette transporter genes. Biochim Biophys Acta 1775: 237-262, 2007

5. Broadley KW, Hunn MK, Farrand KJ, Price KM, Grasso C, Miller RJ, Hermans IF and McConnell MJ: Side population is not necessary or sufficient for a cancer stem cell phenotype in glioblastoma multiforme.Stem Cells 29: 452-461, 2011.

6. Van den Broeck A, Vankelecom H, Van Delm W, Gremeaux L, Wouters J, Allemeersch J, Govaere O, Roskams T and Topal B: Human pancreatic cancer contains a side population expressing cancer stem cell-associated and prognostic genes. PLoS One 8 e73968, 2013.

7. Huang B, Huang YJ, Yao ZJ, Chen X, Guo SJ, Mao XP, Wang DH, Chen JX and Qiu SP: Cancer stem cell-like side population cells in clear cell renal cell carcinoma cell line 769P. PLoS One 8: e68293, 2013.

8. Hiraga T, Ito $\mathrm{S}$ and Nakamura $\mathrm{H}$ : Side population in MDAMB-231 human breast cancer cells exhibits cancer stem cell-like properties without higher bone-metastatic potential. Oncol Rep 25: 289-296, 2011

9. Guo D, Xu BL, Zhang XH and Dong MM: Cancer stem-like side population cells in the human nasopharyngeal carcinoma cell line CNE-2 possess epithelial mesenchymal transition properties in association with metastasis. Oncol Rep 28: 241-247, 2012.

10. Golebiewska A, Brons NH, Bjerkvig R and Niclou SP: Critical appraisal of the side population assay in stem cell and cancer stem cell research. Cell Stem Cell 8: 136-147, 2011.

11. Kato K: Stem cells in human normal endometrium and endometrial cancer cells: Characterization of side population cells Kaohsiung J Med Sci 28: 63-71, 2012.

12. Liu F, Liu D, Yang Y and Zhao S: Effect of autophagy inhibition on chemotherapy-induced apoptosis in A549 lung cancer cells. Oncol Lett 5: 1261-1265, 2013.
13. Ojha R, Jha V, Singh SK and Bhattacharyya S: Autophagy inhibition suppresses the tumorigenic potential of cancer stem cell enriched side population in bladder cancer Biochim Biophys Acta 1842: 2073-2086, 2014.

14. O'Connor ML, Xiang D, Shigdar S, Macdonald J, Li Y, Wang T, Pu C, Wang Z, Qiao L and Duan W: Cancer stem cells: A contentious hypothesis now moving forward. Cancer Lett 344: 180-187, 2014.

15. Fitzgerald TL and McCubrey JA: Pancreatic cancer stem cells: Association with cell surface markers, prognosis, resistance, metastasis and treatment. Adv Biol Regul 56: 45-50, 2014.

16. Kaur S, Singh G and Kaur K: Cancer stem cells: An insight and future perspective. J Cancer Res Ther 10: 846-852, 2014.

17. Mannello F: Understanding breast cancer stem cell heterogeneity: Time to move on to a new research paradigm. BMC Med 11: 169, 2013.

18. Garraway IP: Will identification of a prostate cancer stem cell lead to its cure? Urol Oncol 30: 351-352, 2012.

19. Gespach C: Stem cells and colon cancer: The questionable cancer stem cell hypothesis. Gastroenterol Clin Biol 34: 653-661, 2010.

20. Chandrasekaran S and DeLouise LA: Enriching and characterizing cancer stem cell sub-populations in the WM115 melanoma cell line. Biomaterials 32: 9316-9327, 2011.

21. Rahman M,Deleyrolle L, Vedam-Mai V, Azari H, Abd-El-Barr M and Reynolds BA: The cancer stem cell hypothesis: Failures and pitfalls. Neurosurgery 68: 531-545, 2011.

22. Sellheyer K: Basal cell carcinoma: Cell of origin, cancer stem cell hypothesis and stem cell markers. Br J Dermatol 164: 696-711, 2011.

23. Hadnagy A, Gaboury L, Beaulieu R and Balicki D: SP analysis may be used to identify cancer stem cell populations. Exp Cell Res 312: 3701-3710, 2006.

24. Nishida $\mathrm{S}$ and Masumori N: Cancer stem cells in prostate cancer. Nihon Rinsho 72: 2229-2233, 2014 (In Japanese).

25. Blacking TM, Waterfall M and Argyle DJ: CD44 is associated with proliferation, rather than a specific cancer stem cell population, in cultured canine cancer cells. Vet Immunol Immunopathol 141: 46-57, 2011.

26. Meijer AJ and Codogno P: Autophagy: Regulation by energy sensing. Curr Biol 21: R227-R229, 2011.

27. Hwang MS and Baek WK: Glucosamine induces autophagic cell death through the stimulation of ER stress in human glioma cancer cells. Biochem Biophys Res Commun 399: 111-116, 2010.

28. Mathew R and White E: Autophagy in tumorigenesis and energy metabolism: Friend by day, foe by night. Curr Opin Genet Dev 21: 113-119, 2011. 